\title{
Hubble Diagram Test of 280 Supernovae Redshift Data
}

\author{
Laszlo A. Marosi \\ Paul-Klee-Str.5, D-67061 Ludwigshafen am Rhein, Germany \\ Email: LaszloMarosi@aol.com
}

Received November 9, 2013; revised December 7, 2013; accepted January 2, 2014

Copyright (C) 2014 Laszlo A. Marosi. This is an open access article distributed under the Creative Commons Attribution License, which permits unrestricted use, distribution, and reproduction in any medium, provided the original work is properly cited. In accordance of the Creative Commons Attribution License all Copyrights (C) 2014 are reserved for SCIRP and the owner of the intellectual property Laszlo A. Marosi. All Copyright (C) 2014 are guarded by law and by SCIRP as a guardian.

\section{ABSTRACT}

We compare the Hubble diagram calculated from the observed redshift (RS)/magnitude $(\mu)$ data of 280 Supernovae in the RS range of $z=0.0104$ to 8.1 with Hubble diagrams inferred on the basis of the exponential tired light and the Lambda Cold Dark Matter ( $\Lambda$ CDM) cosmological model. We show that the experimentally measured Hubble diagram follows clearly the exponential photon flight time $\left(t_{S}\right) / \mathrm{RS}$ relation, whilst the data calculated on the basis of the $\Lambda$ CDM model exhibit poor agreement with the observed data.

\section{KEYWORDS}

\section{Redshift/Magnitude Data Fitting; Linear Hubble Relation; Exponential Hubble Relation; $\Lambda$ CDM Cosmological Model}

\section{Introduction}

The basic assumption of the Lambda Cold Dark Matter $(\Lambda \mathrm{CDM})$ cosmological model is that the universe is expanding, according to the Hubble's law [1], at a velocity of $v=z c=H_{0} D_{C}$, where $z$ is the redshift (RS), $c$ is the velocity of light, $H_{0}$ is the Hubble constant, and $D_{C}$ is the co-moving radial distance that can be derived from the observable $z / \mu$ data by (1).

$$
D_{C}=\frac{10^{\frac{\mu+5}{5}}}{(z+1)} \times 3.085 \times 10^{18}
$$

An important test of confidence in modeling the universal expansion is to compare the observed $z / \mu$ data with those derived on the basis of the $\Lambda \mathrm{CDM}$ model. The results presented in the literature, however, are not undisputed and are still a matter of debate. LaViolette [2] and more recently, López-Corredoira [3], Crawford [4], and Marosi [5-7] have shown that the static or slowly expanding universe models fit the observational data better than the data calculated on the basis of the presently prevailing $\Lambda$ CDM model.

Such results, however, are usually refuted with the ar- gument that the static universe contradicts many other cosmological observations, for example, the time dilation test and the cosmic microwave background (CMB) temperature versus RS test [8].

It is not the aim of this paper to argue in favor of or against either the expanding or static cosmological models. We only want to examine which of the two relations: the linear Hubble's law or the exponential $e^{H_{0} \frac{D_{c}}{c}}=z+1$ fits the observational RS/ $\mu$ data more accurately.

We mean that the result of a proper data fitting procedure of reliable observational data cannot be ignored out of respect to the predictions of a theory. If facts contradict the theoretical expectations, then the only scientifically adequate answer can be that the underlying theory is at best, incomplete.

In this paper, we analyze the observed Hubble diagram compiled from 280 supernovae $z / \mu$ data in the range of $z$ $=0.0104$ to 8.1. We expect that in the high RS range, it should be possible to check more precisely whether the Hubble diagram follows a linear $z=H_{0} D_{C} / c$ relation, or the exponential

$$
e^{H_{0} \frac{d}{c}}=e^{H_{0} t_{s}}=z+1
$$


relation; an effect that is perceptible only slightly in the $z$ $<1$ region.

\section{Data Collection and Processing}

In our analysis, we have included 171 gold-set data [9], 59 calibrated high-RS gamma-ray burst (GRB) data (Hymnium data set) and 50 low-RS GRBs obtained by Wei [10] from the 557 Union 2-compilation.

As the $z / \mu$ data are plagued by considerable scatter, similar to the procedure described in [5], the potential $\mu=$ $a \times z^{b}$ function was used to perform a global fitting over the RS range of $z=0.0104$ to 8.1.

As differences between the different cosmological models become more pronounced only in the linear $t_{S} / z$ data representation, using Equations (2)-(4), the potential best fit data were converted into a $t_{s} / z$ data set.

The photon flight time $t_{S}$ was calculated from

$$
t_{s}=\frac{D_{c}}{c}=\frac{10^{\frac{\mu+5}{5}}}{(z+1) \times 3 \times 10^{10}} \times 3.085 \times 10^{18}
$$

In Equations (2) and (3), $t_{S}$ means the flight time of the photons from the co-moving radial distance $D_{C}$ to the observer, which should not be confused with the photon travel time $(t)$ in an expanding universe. $t_{S}$ means the flight time of photons between emission and reception, $\left(t_{S}\right.$ $=D_{C} / c, c$ is the velocity of light), which is proportional to the $D_{C}$ that in entered in the linear Hubble law.

The photon flight time $t_{S}$ for the $\Lambda \mathrm{CDM}$ model was calculated with $H_{0}=72.6 \mathrm{~km} \cdot \mathrm{s}^{-1} \cdot \mathrm{Mpc}^{-1}, \Omega_{\mathrm{M}}=0.266, \Omega_{\Lambda}$ $=0.732$ and $k=0$ [11].

For the purpose of performing $\chi$-squared tests in the high RS range of $t_{S} \times 10^{-14}=6000$ to 11000 between the potential best fit and the $t_{S} / z$ data calculated on the basis of the $\Lambda \mathrm{CDM}$ model we included 41 equidistant $t_{S} / z$ data points in addition to the observed data.

The dimension of $H_{0}$ for the exponential function is expressed by the energy loss with time and it has the dimension $\mathrm{Hz} \cdot \mathrm{s}^{-1} \cdot \mathrm{Hz}^{-1}$ instead of $\mathrm{km} \cdot \mathrm{s}^{-1} \cdot \mathrm{Mpc}^{-1}$ as in the $\Lambda \mathrm{CDM}$ model.

Excel, Excel Solver and WinSTAT [12] software were used for the data fitting, refinement, and analysis and data presentation.

\section{Results}

The potential best fit curve of the 280 observed $z / \mu$ data points is shown in Figure 1.

Four outliers with standard deviation $>3 \sigma$ were identified in the $z / \mu$ data set and omitted from further regression analysis.

Results are shown in Tables 1 and 2.

It can be seen from Table 2 that the omitted outliers have relatively little influence on the regression coeffi-

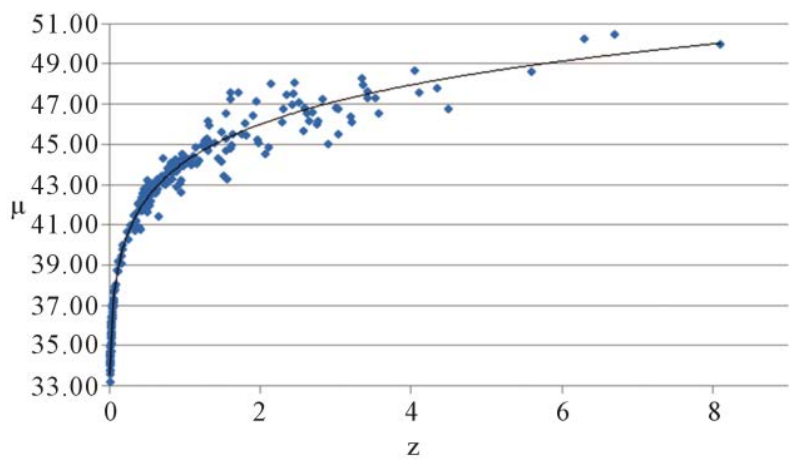

Figure 1. Solid line: potential $\mu=a \times z^{b}$ fit, diamonds: observed $\mathrm{RS} / \mu$ data.

Table 1. Outliers in the regression with $\mathbf{2 8 0}$ data points.

\begin{tabular}{cccc}
\hline Row & $z$-value & $\mathrm{n} *$ Sigma & $\mathrm{P}<0.05$ \\
\hline 277 & 5.6 & 3.981 & 0.0152 \\
278 & 6.29 & 4.573 & 0.0009 \\
279 & 6.695 & 4.921 & 0.0001 \\
280 & 8.1 & 6.127 & $6.218 \mathrm{E}-8$ \\
\hline
\end{tabular}

Table 2. Results of regression with $\mu=a \times z^{b}$ using 171, 276 and $280 \mathrm{z} / \mu$ data points.

\begin{tabular}{cccccc}
\hline Data points & $\mathrm{a}$ & $\mathrm{b}$ & $\mathrm{R} 2$ & $\sum \chi_{\text {square }}$ & $\mathrm{P}_{\chi \text { test }}, \mu_{\text {obs }} / \mu_{\text {calc }}$ \\
\hline 171, Ref.[5] & 44.102 & 0.0593 & 0.9571 & 1.96634 & 1 \\
276 & 44.109769 & 0.059883 & 0.9843 & 1.95407 & 1 \\
280 & 44.1201 & 0.060005 & 0.9871 & 1.95407 & 1 \\
\hline
\end{tabular}

cients $\mathrm{a}$ and $\mathrm{b}$ and that all the results for $\mathrm{a}$ and $\mathrm{b}$ lie within the very small error limits of $a \pm 0.02$ and $b \pm 0.0006$, respectively.

For further data treatment the potential best fit function obtained from 276 data points

$$
\mu=44.109769 \times \mathrm{z}^{0.059883}
$$

was used.

Tables 3-6 show the statistics of the fitting procedure with 276 data points.

\section{The $t_{S} /(z+1)$ Data Representation}

Figure 2 shows the Hubble diagrams measured and calculated with $e^{2.024 \times 10^{-18} \times t_{s}}=z+1$ in the range of $z+1=$ 1.0104 to 5.35 .

The goodness of fit indicators between the observed $t_{S} /(z+1)$ data and the exponential $e^{2.024 x}$ function for $z+$ $1=1.0104$ to 5.5, 6.5 and 9.1 are summarized in Table 7. The precise agreement between the measured and calculated data in the range of $z+1=1.0104$ to 5.5 strongly supports the conclusion that the $t_{S} /(z+1)$ function is exponential. It seems very likely that the small deviations at $\mathrm{z}$ $+1>5.5$ are due to small systematic errors in distance measurements or to the calibration method at very high RSs. 
Table 3. Descriptive statistics $\boldsymbol{\mu} / \mathbf{z}$.

\begin{tabular}{|c|c|c|c|c|c|c|c|}
\hline \multirow{3}{*}{$\mu$} & \multirow{3}{*}{$\begin{array}{c}\text { Valid cases } \\
276 \\
\text { Variation coefficient }\end{array}$} & \multirow{3}{*}{$\begin{array}{c}\text { Mean } \\
41.76894928 \\
\text { Rel. V. coefficient (\%) }\end{array}$} & \multirow{2}{*}{\multicolumn{2}{|c|}{$\begin{array}{c}\text { Std. error of mean } \\
0.242237842\end{array}$}} & \multirow{3}{*}{$\begin{array}{r}\text { Variance } \\
16.19545144 \\
\text { Minimum }\end{array}$} & \multirow{2}{*}{\multicolumn{2}{|c|}{$\begin{array}{c}\text { Std. Deviation } \\
4.02435727\end{array}$}} \\
\hline & & & & & & & \\
\hline & & & Skew & Kurtosis & & Maximum & Range \\
\hline$\mu$ & 0.096348061 & 0.57994 & -0.622585385 & -0.735770108 & 33.21 & 48.68 & 15.47 \\
\hline
\end{tabular}

Table 4. Descriptive statistics $z / \mu$.

\begin{tabular}{|c|c|c|c|c|c|c|c|}
\hline \multirow{3}{*}{$z$} & \multirow{3}{*}{$\begin{array}{c}\text { Valid cases } \\
276 \\
\text { Variation coefficient }\end{array}$} & \multirow{3}{*}{$\begin{array}{c}\text { Mean } \\
0.880278623 \\
\text { Rel. V. coefficient (\%) }\end{array}$} & \multirow{2}{*}{\multicolumn{2}{|c|}{$\begin{array}{c}\text { Std. error of mean } \\
0.056577708\end{array}$}} & \multirow{3}{*}{$\begin{array}{c}\text { Variance } \\
0.883486217 \\
\text { Minimum }\end{array}$} & \multirow{2}{*}{\multicolumn{2}{|c|}{$\begin{array}{c}\text { Std. Deviation } \\
0.939939475\end{array}$}} \\
\hline & & & & & & & \\
\hline & & & Skew & Kurtosis & & Maximum & Range \\
\hline$z$ & 1.067774964 & 6.427249997 & 1.581919841 & 2.212059268 & 0.0104 & 4.5 & 4.4869 \\
\hline
\end{tabular}

Table 5. Variable: $\boldsymbol{\mu}$; grouped by z; $95 \%$ confidence level.

\begin{tabular}{cccccc}
\hline$\mu$ & $N$ & Mean & Conf. $( \pm)$ & Std. Error & Std. Dev. \\
\hline 33 to 34 & 4 & 0.012725 & 0.004668869 & 0.01467069 & 0.002934138 \\
34 to 35 & 21 & 0.016414286 & 0.001003628 & 0.000481134 & 0.002204832 \\
35 to 36 & 18 & 0.027077778 & 0.001658866 & 0.000786261 & 0.0003335823 \\
36 to 37 & 15 & 0.41213333 & 0.003964023 & 0.001848213 & 0.007158099 \\
37 to 38 & 10 & 0.0625 & 0.006904831 & 0.003052322 & 0.009652288 \\
38 to 39 & 3 & 0.104666667 & 0.046628617 & 0.010837179 & 0.018770544 \\
39 to 40 & 5 & 0.1612 & 0.027294023 & 0.009830565 & 0.021981811 \\
40 to 41 & 6 & 0.330166667 & 0.077409426 & 0.030113581 & 0.073762908 \\
41 to 42 & 16 & 0.42775 & 0.050113435 & 0.023511433 & 0.094045734 \\
42 to 43 & 46 & 0.521336957 & 0.33007283 & 0.016388078 & 0.111149352 \\
43 to 44 & 43 & 0.813906977 & 0.061365095 & 0.030407637 & 0.199396209 \\
44 to 45 & 37 & 1.167456757 & 0.103807992 & 0.0511849998 & 0.311346189 \\
45 to 46 & 15 & 1.823953333 & 0.321476572 & 0.149887432 & 0.580511527 \\
46 to 47 & 19 & 2.640421053 & 0.355072924 & 0.169008137 & 0.73668939 \\
47 to 48 & 14 & 2.799142857 & 0.529540171 & 0.245115646 & 0.917138766 \\
48 to 49 & 4 & 2.99875 & 1.37869082 & 0.4332173 & 0.8664346 \\
Entire sample & 276 & 0.880278623 & 0.111380452 & 0.056577708 & 0.939939475 \\
\hline
\end{tabular}

Table 6. Variable: $z$; grouped by $\boldsymbol{\mu}$; $95 \%$ confidence level.

\begin{tabular}{cccccc}
\hline$z$ & $N$ & Mean & Conf. $( \pm)$ & Std. Error & Std. Dev. \\
\hline 0.0 to 0.5 & 120 & 38.1235 & 0.578568683 & 0.292191652 & 3.200799178 \\
0.5 to 1.0 & 76 & 3.29763158 & 0.148926165 & 0.074758293 & 0.65172769 \\
1.0 to 1.5 & 28 & 44.6478571 & 0.233356837 & 0.113731045 & 0.601808122 \\
1.5 to 2.0 & 18 & 45.6505555 & 0.62841576 & 0.297853401 & 1.263684958 \\
2.0 to 2.5 & 9 & 46.6894444 & 1.000358877 & 0.433806188 & 1.301418564 \\
2.5 to 3.0 & 10 & 46.331 & 0.485711017 & 0.214711434 & 0.678977172 \\
3.0 to 3.5 & 9 & 46.975 & 0.693006582 & 0.300522693 & 0.901568078 \\
3.5 to 4.0 & 2 & 46.93 & 4.828358221 & 0.38 & 0.537401154 \\
4.0 to 4.5 & 3 & 48.0166666 & 1.45068823 & 0.3371611353 & 0.583980593 \\
4.5 to 5.0 & 1 & 46.74 & - & - & - \\
Entire sample & 276 & 41.7689492 & 0.476876166 & 0.242237842 & 4.02435727 \\
\hline
\end{tabular}


Table 7. Goodness of fit indicators.

\begin{tabular}{ccccccc}
\hline Data points & $z+1$ range & $\mathrm{R}^{2}$ & Std. error & Std. dev. & $\sum \chi^{2}$ & $\mathrm{P}$ \\
\hline 276 & $1.0104-5.5$ & 0.99996 & 0.006190 & 0.933469 & 0.007723956 & 1 \\
278 & $1.0104-6.5$ & 0.99985 & 0.019795 & 1.027633 & 0.010656491 & 1 \\
280 & $1.0104-9.1$ & 0.99838 & 0.046957 & 1.173129 & 0.088924843 & 1 \\
\hline
\end{tabular}

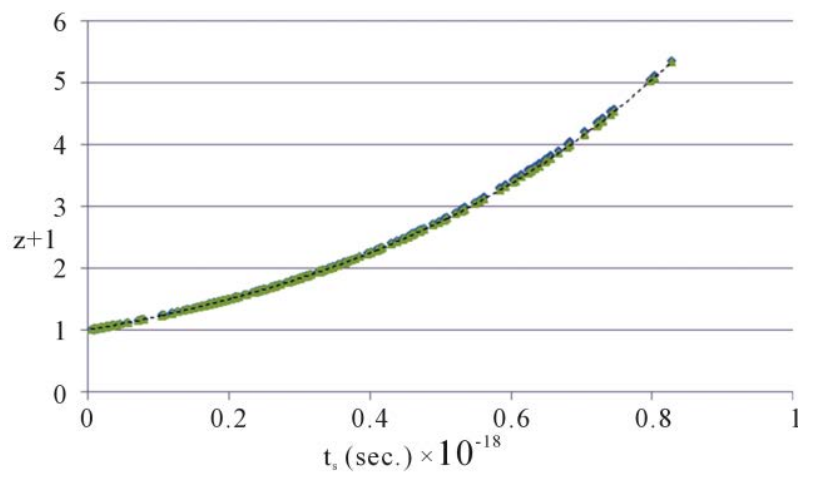

Figure 2. Observed data (diamonds), data calculated with $e^{2.024 \times 10^{-18} \times t_{s}}$ (triangles), trendline with $e^{2.024 x}$ (dashed line).

\section{$t_{S} / z$ Diagram in the Range of $z=0.0104$ - 8.1, Comparison with the $\Lambda$ CDM Model}

Figure 3 shows the $t_{S} / z$ diagram in the range of $z=$ 0.0104 to 8.1 calculated using Equation (4) with the observed $z / \mu$ data set (squares), the exponential function $z=e^{2.024 \times 10^{-18} \times t_{s}}-1$ (triangles), and the $t_{S} / z$ relation derived from the $\Lambda$ CDM model (circles) with $H_{0}=72.6 \mathrm{~km}$ $\mathrm{s}^{-1} \mathrm{Mpc}^{-1}, \Omega_{\mathrm{M}}=0.266, \Omega_{\Lambda}=0.732$ and $k=0$.

One can see from Figure 3 that, similar to the plot shown in Figure 2, the curves calculated from the best fit and the exponential function $z=e^{2.024 \times 10^{-18} \times t_{s}}-1$ are nearly concurrent over the entire range of $z$, Pchi square $=1$, whilst at $z>2$ the $t_{S} / z$ data calculated based on the $\Lambda \mathrm{CDM}$ model show clearly a different slope and depart considerably from both, the linear and the exponential functions. The $\chi$-square test indicates statistical significance between the observed $t_{S} / \mu$ and the calculated $\Lambda \mathrm{CDM}$ data of $\mathrm{P}=0.0173$, indicating that from a statistical point of view, the two models are essentially different.

At RSs $z<0.3$ (Figure 4 ), the $t_{S} / z$ curves for the potential best fit, the exponential function, and the $\Lambda \mathrm{CDM}$ model can be fitted with the linear function $\mathrm{z}=$ $0.000228725 \times t_{S}-0.00332331\left(\mathrm{R}^{2}=0.9989\right)$ with good approximation. The linear approximation, however, is deceiving. As can be seen in Figure 3, that at high RSs, the best-fit and the exponential curves follow strictly the exponential energy depletion relationship.

\section{Conclusions}

The most impressive result of the Hubble diagram test is

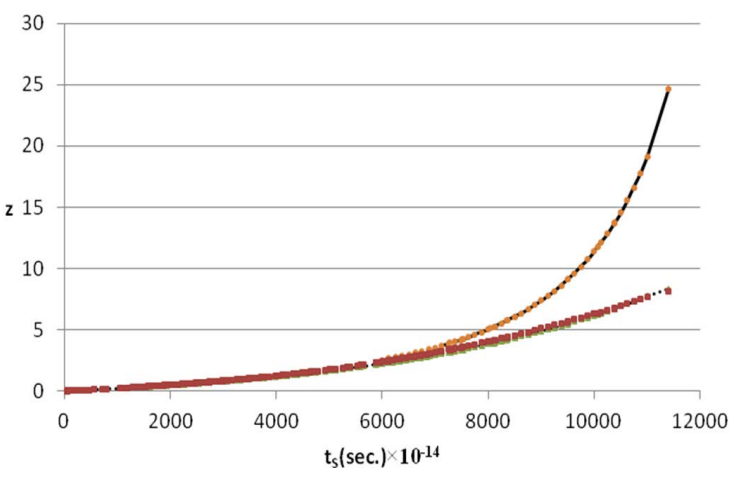

Figure 3. Redshift of type Ia supernovae as a function of $\boldsymbol{t}_{\mathrm{s}}=$ $D_{C} / c$. Squares: $t_{S} / z$ data inferred from the potential best-fit curve of the observed $z / \mu$ diagram. Triangles: the exponential $t_{S} / z$ relation with $H_{0}=2.024 \times 10^{-18}$. Circles: $t_{S} / z$ relation derived from the $\Lambda \mathrm{CDM}$ model with $H_{0}=72.6$ $\mathbf{k m} \cdot \mathbf{s}^{-1} \cdot \mathbf{M p c}^{-1}$.

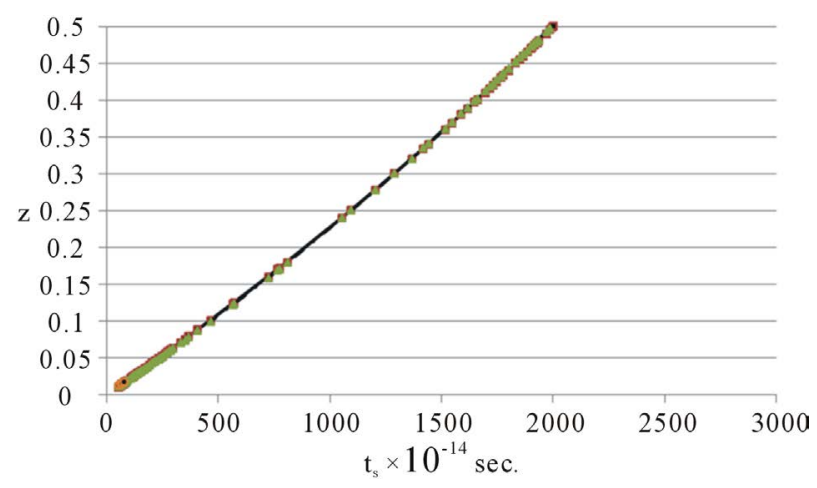

Figure 4. The "linear" $t_{S} / z$ relation in the low RS region for the potential best fit and the exponential function, and for the $t_{S} / z$ data calculated based on the $\Lambda$ CDM model.

that the $t_{S} / z$ relation obtained from the potential best fit data can be expressed nearly exactly by the exponential formula $e^{2.024 \times 10^{-18} t_{s}}=z+1$ over the entire range of $z=$ 0.01 to 8.1 .

In contrast, in the RS range $z>2$ the $t_{S} / z$ curve derived from the $\Lambda \mathrm{CDM}$ model with $H_{0}=72.6 \mathrm{~km} \cdot \mathrm{s}^{-1} \cdot \mathrm{Mpc}^{-1}, \Omega_{\mathrm{M}}$ $=0.266, \Omega_{\Lambda}=0.732$ and $k=0$, shows poor agreement with the observed data. The $\chi$-square test indicates statistical significance between the observational potential fit and the calculated $\Lambda \mathrm{CDM}$ data of $\mathrm{P}=0.0173$, indicating that from a statistical point of view the two models are essentially different.

Based on the results presented in this paper, a reconsideration of the $\Lambda \mathrm{CDM}$ model appears warranted. 


\section{Acknowledgements}

I am grateful to Professor Rainer Mattes of the Westfälische Wilhelms-Universität, Münster, Germany, for his support and his continuous interest in this work..

\section{REFERENCES}

[1] E. P. Hubble, Proceedings of the National Academy of Sciences of the United States of America, Vol. 15, 1929, pp. 167-173. http://dx.doi.org/10.1073/pnas.15.3.168

[2] P. A. LaViolette, The Astrophysical Journal, Vol. 301, 1986, pp. 544-553. http://dx.doi.org/10.1086/163922

[3] M. López-Corrediora, Recent Research in Development of Astronomy \& Astrophysics, Vol. 1, 2003, p. 561. http://arxiv.org/abs/astro-ph/0310214

[4] D. A. Crawford, The Journal of Cosmology, Vol. 13, 2011, pp. 3875-3946.

[5] L. A. Marosi, Advances in Astronomy, Vol. 2013, 2013, Article ID: 917104. http://dx.doi.org/10.1155/2013/917104

[6] L. A. Marosi, "Hubble Diagram Test of Expanding and Static Cosmological Models, A New Interpretation of the
Cosmic Redshift," The General Science Journal, Research Papers-Astrophysics/Download/4931, 18 June 2013. http://gsjournal.net/Science-Journals/Essays-Astrophysics /Download/4931

[7] L. A. Marosi, Physics Research International, Vol. 2012, 2012, Article ID: 640605. http://dx.doi.org/10.1155/2012/640605

[8] A. Sandage, "The Universe at Large. Key Issues in Astronomy and Cosmology,” Cambridge University Press, Cambridge, 1992.

[9] A. G. Riess, A. G. Sirolger, J. Tonry, et al., The Astrophysical Journal, Vol. 602, 2004, pp. 665-687. http://dx.doi.org/10.1086/383612

[10] H. Wei, Journal of Cosmology and Astroparticle Physics, Vol. 2010, 2010, Article ID: 020. http://dx.doi.org/10.1088/1475-7516/2010/08/020

[11] E. L. Wright, Publications of the Astronomical Society of the Pacific, Vol. 118, 2006, pp. 1711-1715. http://dx.doi.org/10.1086/510102

[12] WinSTAT, “Statistik-Add-In für Microsoft ${ }^{\circledR}$ Excel,” R. Fitch Software, 2013. http://www.winstat.de 